\title{
Interactive comment on "Source apportionment of carbonaceous chemical species to fossil fuel combustion, biomass burning and biogenic emissions by a coupled radiocarbon-levoglucosan marker method" by Imre Salma et al.
}

\section{Anonymous Referee \#1}

Received and published: 5 July 2017

The paper "Source apportionment of carbonaceous chemical species to fossil fuel combustion, biomass burning and biogenic emissions by a coupled radiocarbonlevoglucosan marker method" by Salma et al. aims at proposing a source apportionment methodology based on simultaneous measurements of $14 \mathrm{C}$ in the total carbon (TC) fraction of carbonaceous aerosol and levoglucosan measurements.

The approach can be of interest for the scientific community. The source apportionment approach proposed in the paper, joining 14C measurements on TC and levoglucosan determination, can provide information similar to those obtained by $14 \mathrm{C}$ measurements 
in $\mathrm{OC}$ and EC - which are still performed by few groups in the world - even if further a-priori assumption on (LVG/EC)BB emission ratio is needed. Nevertheless, different criticalities are present in the current version of the manuscript. Thus, the paper needs to be thoroughly revised (major revision) before being considered for publication on ACP.

The main aspects requiring attention are the following:

- source apportionment scheme description should be re-written to better clarify the information flow. Figure 3 has to be completely revised;

- indication on source apportionment uncertainties is mostly missing, some hints for their estimate should be added to the text; furthermore, possible role of secondary organic compounds on OC-BIO source apportionment needs further comments;

- assumptions needed for the application of the approach should be stated more clearly;

- high time resolved measurements of carbonaceous fractions and aerosol absorption/extinction properties were performed in parallel to filter sampling, but they are rarely considered in data analysis. I suggest the authors to consider one of the following possibilities: a) completely remove the description of techniques and the introduction on paragraph 3; b) revise optical properties data (currently, data treatment is not adequate, see detailed comments) and exploit multi-wavelength measurements to attempt optical source apportionment approaches, i.e. Aethalometer model (Sandradewi et al., 2008 Environ. Sci. Technol. 42, 3316-3323 and following modifications) or Multi-Wavelength Absorption Analyser model (Massabò et al., 2015. Atm. Environ, $108,1-12)$. These models provide information on fossil fuel combustion/biomass burning contribution to carbonaceous aerosol basing on optical absorption measurements and thermal-optical OC/EC/TC. The information could support the results obtained by the $14 \mathrm{C}$ on TC and LVG source apportionment proposed in the paper. 
- page 4, par. 2.2: indication on minimum detection limits and uncertainty for the mentioned techniques are completely missing. Please, add such information (a table should be enough)

- page 5, line 37. The authors used the same fm reference for modern material (1.08), independently on the source (BB or BIO). Usually in $14 \mathrm{C}$ source apportionment studies, two different values are chosen for BB (expected higher due to integrated signal) and $\mathrm{BIO}$ (which is expected to be in equilibrium with today atmospheric $14 \mathrm{C} / 12 \mathrm{C}$ ratio. The approximation should be highlighted (see e.g. par 2.4 Minguillòn et al., 2011 cited by the authors)

- page 6, line 2: FDMS usually operates at $30^{\circ} \mathrm{C}$. Any comment about possible mass losses (e.g. nitrate?)

The following two comments are of interest only if the authors decide not to remove the part concerning high time resolved optical and thermal-optical measurements

- page 6, line 6 and 9-10. Basing on Drinovec et al. (2015), Atmos. Meas. Tech., 8, 1965-1979 (page 1970) I guess that $16.6 \mathrm{~m} 2 / \mathrm{g}$ is the mass extinction (not absorption) coefficient: Aethalometer assumes proportionality between babs and batn through a proportionality factor C - Weingartner et al, (2003), J. Aerosol Sci., 34, 1445-1463. The mass absorption coefficient historically considered by Aethalometer @880 nm is $7.77 \mathrm{~m} 2 / \mathrm{g}$ (Drinovec et al. (2015)). If a-priori assumption has to be applied to convert PAS measurements into $\mathrm{BC}$ concentration maintaining equivalence with what performed in the Aethalometer, then $7.77 \mathrm{~m} 2 / \mathrm{g}$ is the value to be extrapolated to $1064 \mathrm{~nm}$ for application to PAS measurements (please evaluate also the following comment).

- page 6, lines 18-23: the comparisons between optical EBC (from AE or PAS) and thermal EC are strongly affected by the assumption on absorption/extinction coefficients 
chosen a-priori. As such values are site/season/composition dependent, the parallel availability of optical (batn/babs) and thermal (EC) measurements could be better exploited to provide information on BC mass absorption coefficients in Budapest;

- page 7, line 37-38: in the cited works, SFUs are reported to have a cut-off diameter of $2 \mu \mathrm{m}$. Did the authors change e.g. nuclepore filter pore size or flow-rate to modify the size cut? Different cut-off size could partially justify differences, as well as the use of different instrumentation (i.e. SFU vs. TEOM) or the improvement of combustion technologies in the last 15 years, leading to smaller particles formation.

- page 10, lines 9-15. Lignite contribution and its high LVG/MAN does not allow reliabile softwood/hardwood quantification. I suggest to completely remove this paragraph.

- page 11, line 27: "the $\mathrm{fC}$ values of the back filters were individually taken into account for the front filters". Please better explain the procedure for front filter correction.

- page 12, line 5: from Bernardoni et al. (2013) I guess the mentioned value was obtained elsewhere (Bernardoni et al, 2011, Sci Total Environ, 409, 4788-4795)

- page 12, figure 3 and lines 9-14: I don't think the chart correctly represents information flow described in the text. Chart information flow makes argue that factor $\mathrm{f} 2$ and $\mathrm{f} 3$ are needed to determine EC-BB and OC-BB from TC-C and OC-C. This interpretation would be nonsense, as no proportionality is expected between EC-BB and TC-C, as well as between OC-BB and OC-C (due to at least an independent source - BIO - contributing to $\mathrm{OC}-\mathrm{C}$ ). Nevertheless, the text shows that the source apportionment follows a completely different path. If I correctly interpreted the text, it should be evidenced in the chart that input data are obtained from 14C information (TC-C), and LVG (OC-BB and EC-BB thanks to emission ratios). $\mathrm{f} 2$ and $\mathrm{f} 3$ are then a by-product (by ratios or subtractions) of these quantities and do not have anything to do with "mass balance equations", opposite to what reported at line 10. Then, I guess from the text that EC-FF 
is derived as difference between total EC and EC-BB. EC-FF is then exploited to obtain the factor $\mathrm{f} 4$. I suggest to completely re-think the chart, including external inputs and their role in the source apportionment scheme. Arrows should start from input data and point to calculated quantities. Furthermore, corresponding text (lines 9-14, page 12 ) is quite obscure in its current form, and it should be re-written to better evidence the real information flow.

Interactive

comment

- page 12, lines 14-17: I suggest to mention also a disadvantage of the proposed model compared to the direct determination of $14 \mathrm{C}$ in $\mathrm{OC}$ and $\mathrm{EC}$ fraction, mainly impacting on EC source apportionment: in the case of the proposed model, EC source apportionment requires assumption on wood burning LVG/EC emission ratio, whereas if $14 \mathrm{C}$ is directly measured in the EC fraction, the apportionment is straightforward.

- Page 13, line 25: "The importance of BB sources, FF combustion and biogenic sources for the PM2.5 mass". Please note that the model does not apportion the total contribution of these sources to PM2.5, but only the contribution of carbonaceous species emitted by these sources (e.g. high emissions of $\mathrm{K}+$ from BB combustion are expected to impact on PM2.5, but they are - of course - completely neglected by this source apportionment approach). Their total relative impact to PM2.5 is for sure higher, and the proportions among them can be different.

- Page 13, line 31: please, add some considerations on estimated uncertainties related to the model. Page 14, line 16: please add considerations on the possible impact of secondary compounds related to BB emission on OC-BIO estimates

- Page 14, line 19 and lines 26-27: at line 19 the authors find correlations between NOx (assumed as markers for traffic emissions) and EC-FF, whereas no significant correlation is found between NOx and OC-FF. I think this observation supports considerations 2 and 3 at lines 26, 27. Nevertheless, an important role can be played also by secondary OC-FF, justifying disentanglement between primary species from FF combustion and OC-FF. It should also be noticed that to avoid effects due to differ-

Printer-friendly version

Discussion paper 
ent repartition of $\mathrm{NO}$ vs. NO2, NOx concentration should be better represented in ppb for the evaluation of more robust correlation coefficients.

Minor comments - page 3, line 39: what is TEOM size-cut? - page 4, line 8-9: don't agree with the sentence. EUSAAR_2 was initially developed for background sites (see end of introduction in Cavalli et al., 2010 Atmos. Chem. Phys., 11, 10193-10203). Its application has effectively been extended to urban sites. Nevetheless, concerns were posed in the literature on its use in urban areas (e.g. Piazzalunga et al., Atmos. Chem. Phys., 11, 10193-10203, 2011) - page 4, line 11: EUSAAR_2 protocol is longer than 15 minutes. Was it somewhat modified? Please, report, effective protocol - page 4, line 36: why did the authors use different thermal protocols for on-line and off-line instrumentation? - page 8, Table 2: I expected mean values for PM10 being the sum of mean values for PM2.5.and PM10-2.5. It is not so. Is it a time-averaging problem? Or can it be due to non-parallel sampling (e.g. missing values in only one of the two samplers were considered in the average?) - page 9, line 3: LVG is not a substantial part of $O C$ (as can be seen in figure 1). The observation can indicate an important contribution of LVG source (BB) also to other OC species. - page 9, figure 1: please plot $\mathrm{EC}$ and $\mathrm{BC}$ (if maintained) on a secondary axis to better allow appreciating modulations - page 10, line 1: no \% is needed in OC/EC ratio at the beginning of the line. Furthermore, I suggest to comment this datum which gives indication of strong secondary organic aerosol formation. - page 11 line 29: the authors relate the need of sampling artefact correction to the total carbon load on a filter ("1 mg"), and not to the mass/area load. Can they justify this?

Interactive comment on Atmos. Chem. Phys. Discuss., https://doi.org/10.5194/acp-2017-406, 2017.

Interactive

comment

\section{Discussion paper}

